\title{
Fetal Transplant-Induced Restoration of Spatial Memory in Rats with Lesions of the Nucleus Basalis of Meynert
}

\author{
Anthony C. Santucci, Philip D. Kanof and Vahram Haroutunian \\ Psychiatry Service, Bronx Veterans Administration Center \\ 130 West Kingsbridge Road, Bronx, NY 10468 \\ and \\ Dept. of Psychiatry, The Mount Sinai School of Medicine \\ One Gustave L. Levy Place, New York, NY 10029
}

\begin{abstract}
SUMMARY
Bilateral lesions of the nucleus basalis of Meynert (nbM) in rats produced mnemonic deficits when subjects were tested on tests of spatial memory over a period of 3 to 7.5 months postoperatively. The transplantation of cholinergic-rich, fetal ventral forebrain tissue to either two or four frontoparietal cortical sites normalized performance on the spatial memory tasks. However, which transplant condition yielded recovery depended upon the nature of the task and/or posttransplantation interval. When assessed 8 months following transplant surgery, cortical choline acetyltransferase and acetylcholinesterase activity levels in both transplant groups were comparable to those values found in sham-operated animals. These data indicate that fetal transplants can reverse the mnemonic deficits and restore cortical cholinergic neurochemical activity to near-normal levels in rats with nbM lesions.
\end{abstract}

Key words: nucleus basalis of Meynert lesions; spatial memory; fetal transplantation; choline acetyltransferase; acetylcholinesterase.

Reprint address:

Anthony C. Santucci

Psychiatry Service

Bronx Veterans Administration Medical Center

130 West Kingsbridge Road

Bronx, NY 10468

U.S.A.

\section{INTRODUCTION}

An increasing amount of empirical attention is being directed at examining the effects of fetal neural tissue transplantation in animals that have sustained experimentally-induced lesions of the nucleus basalis of Meynert (nbM)-frontal cortical system $/ 9,14 /$. The impetus for these preclinical studies is undoubtedly related to the potential therapeutic role that fetal transplantation may have in treating Alzheimer's disease /8/. A number of such studies have reported cholinergic-rich fetal transplants to be effective in reversing the mnemonic, anatomical and neurochemical consequences of $\mathrm{nbM}$ lesions. For example, in rats with unilateral lesions of the nbM, cortically-placed fetal graphs have been shown to improve passive avoidance and Morris water-maze performance $/ 4 /$ and attenuate cortical choline acetyltransferase activity deficits $/ 6 /$. The anatomical specificity of these effects was suggested by studies showing that fetal nbM tissue, as opposed to fetal septal tissue, produced the greatest amount of fiber outgrowth $15 /$. More recently, cholinergic-rich fetal graphs have been reported to ameliorate the spatial memory impairments produced by bilateral nbM lesions when rats were tested on a delayed spatial alternation task $/ 16 /$. Evidence for anatomical specificity of graph-induced restoration of function was also reported in this study in that cerebellar transplants failed to improve task performance $/ 16 /$.

Despite the demonstration of transplantinduced recovery following nbM lesions, the 
exact parameters necessary for such recovery remain unknown to a large extent. It was in this context that the present study sought to examine several variables that might affect the functional effects of fetal transplants in animals with bilateral nbM lesions. Specifically, the present investigation examined 1) the influence of number of cortical transplant sites on recovery, 2) the generalizability of transplant-induced memory enhancement, and 3) the stability of transplant-mediated functional enhancement. Accordingly, rats prepared with bilateral damage to the $n b M$ were implanted with fetal ventral forebrain tissue at either 0,2 or 4 cortical sites. Subjects were then assessed on a variety of memory tasks over a period of 3 to 7.5 months following transplantation. Eight months after transplantation subjects were sacrificed and their brains examined neurochemically and histologically.

\section{MATERIALS AND METHODS}

\section{Subjects}

Naive Sprague-Dawley adult male rats (250$350 \mathrm{gm}$ ) served as subjects. Subjects were housed in groups of 3-4 in wire-mesh cages and maintained on a $12 \mathrm{hr}$ light/dark cycle (lights on at $7 \mathrm{AM}$ ) with food and water freely available except when noted. All animals underwent lesion, transplantation and/or sham surgery during the same two-week period. The four behavioral tasks were presented to all subjects in the same order and subjects were trained on each task concurrently.

\section{nbM Surgery}

Twenty-six subjects received bilateral ibotenic acid-induced lesions $(5 \quad \mu \mathrm{g} / \quad 1 \quad \mu l \quad 7.4 \quad \mathrm{pH}$ phosphate buffer/side) of the nbM while nine animals underwent sham operations. All subjects were anesthetized with $60 \mathrm{mg} / \mathrm{kg}$ ketamine hydrochloride (im, KETALAR, Parke-Davis, Morris Plains, NJ) and $21 \mathrm{mg} / \mathrm{kg}$ pentobarbital (ip, Sigma, St. Louis, MO) and positioned in a Kopf stereotaxic apparatus with the upper incisor bar level relative to the interaural line. Lesions were performed in a manner identical to the procedure detailed in a previous publication from our laboratory /13/. Infusions of ibotenic acid were made at stereotaxic coordinates -0.3 $\mathrm{mm}$ from bregma; \pm 3.0 from midline; $-8.1 \mathrm{~mm}$ $(0.6 \mu \mathrm{l}),-7.8(0.2 \mu \mathrm{l})$ and $-7.5(0.2 \mu \mathrm{l})$ from skull. For sham operations, the needle was lowered to a depth of $-6.0 \mathrm{~mm}$ and ibotenic acid was not infused.

\section{Fetal Dissection and Transplant Surgery}

Seven to ten days following lesions of the nbM (mean = 9.1 days), fetal tissue was dissected (in the $\mathrm{AM}$ ) and transplanted (in the PM) under sterile conditions according to the general methods of Bjorklund et al. /2/. This entailed dissecting a triangular-shaped piece of fetal ventral forebrain tissue consisting of developing septum-diagonal band/nbM cells from 14 - 16 gestational day-old fetuses (12-16 $\mathrm{mm}$ crown-to-rump length). The caudal border of the extraction was a $4 \mathrm{~mm}$-long incision made immediately rostral to the optic chiasm while the rostral tip was a point at midline just caudal to the olfactory bulbs. Two lateral cuts, each $4 \mathrm{~mm}$ in length, were made just medial to the lateral ventricles and represented the lateral borders of the dissected area. Pieces of fetal tissue were placed in a small sterile glass vial containing $3 \mathrm{ml}$ of Dulbecco's Modified Eagle Medium (DMEM; Sigma) kept at room temperature. Eight to twelve fetuses were dissected over a period of about $45 \mathrm{~min}$. Following dissection of the last fetus, the DMEM solution was removed and replaced with $3 \mathrm{ml}$ of a $0.1 \%$ trypsin (Sigma)/DMEM solution. The tissue was then incubated at $37^{\circ} \mathrm{C}$ for $15 \mathrm{~min}$ followed by thorough washing with DMEM. After washing, $20 \mu 1$ DMEM per dissected fetus were added and the tissue pieces were disrupted by gentle pipetting which resulted in a well-dispersed cell suspension solution.

Transplant surgeries were performed under pentobarbital/ketamine anesthesia. Animals previously prepared with nbM lesions received stereotaxically placed cell suspension implants into the frontoparietal cortices at either two (2TR group) or four (4TR group) sites. The injection volume for each implant site was $1.5 \mu \mathrm{l}$ $\left(2.5 \times 10^{5}\right.$ cells). For the 2TR group $(n=9)$, fetal cell suspension implants were made at stereotaxic coordinates $+3.7 \mathrm{~mm}$ from the 
bregma, $\pm 1.8 \mathrm{~mm}$ lateral from midline, $-2.5 \mathrm{~mm}$ deep from dura. For the 4TR group $(n=8)$, two implants were made at the above coordinates and two implants made at $+1.7 \mathrm{~mm}$ from the bregma, $\pm 2.5 \mathrm{~mm}$ from midline, -2.1 from dura. To ensure that each implant contained a fairly constant concentration of cells, the cell suspension solution was repipetted prior to individual surgeries. Another group of animals with $n b M$ lesions (NBM; $n=9)$, in addition to the sham operated group (SHAM; $n=9$ ), underwent a control surgery which included anesthesia, placement in the stereotaxic apparatus, scalp incision and restitching.

Upon postmortem examination, brains from two animals from each of the two transplant groups were found to contain infections. Infections in these brains apparently interfered with transplant viability, as little or no histological or neurochemical evidence of fetal cell implants was revealed. Therefore, data from these four animals were discarded, reducing the numbers of subjects in the 2TR and 4TR groups to 7 and 6, respectively.

\section{Complex Spatial Discrimination}

Twelve weeks after transplantation or control surgery, subjects were put on a $23 \mathrm{hr}$ water deprivation schedule and trained (1 trial/day, 5 days/week) on a complex spatial discrimination task /12,13/. This spatial memory task required rats to find a water-filled $(2 \mathrm{ml})$ receptacle among a 6 row $\times 5$ column array of receptacles glued to a $122 \times 76 \mathrm{~cm}$ black stone table top. During training all other receptacles remained dry. A choice error was recorded when the animal's snout touched or went below the level of an unbaited receptacle's rim or when the animal searched the baited receptacle but did not drink from it for more than $5 \mathrm{sec}$. The apparatus was wiped clean with a deodorizer (0.01\% Hygeia Odor Control) between animals. Subjects were trained on this task for 20 days and the groups were compared with respect to the number of trials and errors required to reach a criterion of 3 or less errors on 4 out of 5 consecutive days.

\section{Brightness Discrimination}

Immediately following training on the complex spatial discrimination task (i.e., 19 weeks posttransplantation), subjects were trained on a water-motivated brightness discrimination task performed in a black Plexiglas Y-maze /13/. Animals were required to enter the "bright" arm in order to drink water for $30 \mathrm{sec}$ from a spout located at the end of the arm. A similar spout also protruded from the endwall of the "dim" arm but no water could be obtained from this spout. "Bright" arm location was chosen on a random basis with the restriction that either arm could not serve as the "bright" arm on three or more consecutive days. An error was recorded when the animals either 1) entered the "dim" arm with at least three paws, 2) retraced back into the stem with at least three paws or 3) entered the "bright" arm and then exited without drinking for more than $5 \mathrm{sec}$. If an error was committed the animal was allowed to correct itself. The Y-maze was wiped clean with a deodorizer between animals. Subjects were trained ( 1 trial/day, 5 days/week) for 30 days and the groups compared with respect to the number of trials and errors required to reach a criterion of 4 out of 5 consecutive errorless days.

\section{Delayed Spatial Alternation Task}

Twenty-five weeks after transplantation, subjects were trained on a water-motivated delayed spatial alternation task in the Y-maze used above $/ 17 /$. The first daily trial was an informational trial whereby the animal was forced to enter either the right or left arm in order to drink water for $10 \mathrm{sec}$ from the spout protruding from the endwall. On the next trial (i.e., the first choice trial) both arms, each with spouts protruding from the endwalls, were accessible and water was available only in the arm opposite to that used on the informational trial. On subsequent choice trials, both arms remained available. If the animal made a correct choice, the location of the baited arm on the next trial switched. If the animal was incorrect (i.e., 
entered the unbaited arm with at least three paws) an error was recorded and the animal was not given an opportunity to correct itself. On the trials following incorrect choices, the location of the baited arm was not changed. Five choice trials were administered daily with a $30 \mathrm{sec}$ intertrial delay imposed. The Y-maze was wiped clean with a deodorizer between animals. Subjects were trained for 15 days ( 75 choice trials) and groups were compared with respect to the number of trials and errors required to reach a criterion of 16 correct trials on any 20 consecutive trials. Groups were also compared with respect to percent correct (i.e., number of correct trials divided by 75 trials). Three rats from the SHAM group and one rat from the NBM group "froze" and could not be trained. Thus, the numbers of subjects in the SHAM and NBM groups for this task were reduced to 6 and 8 , respectively.

\section{Passive Avoidance}

The final memory task employed was a onetrial, step-through passive avoidance task /15/. Animals were trained on this task 30 weeks posttransplantation. Subjects were put on the white side of a black/white shuttle box and allowed to cross into the black compartment, at which time they received an inescapable 0.6 $\mathrm{mA} / 4 \mathrm{sec}$ electric scrambled footshock. Subjects were confined in the black compartment for 60 sec. Retention was assessed $72 \mathrm{hr}$ later by measuring how quickly (sec) animals crossed from the white compartment into the previously shocked black compartment during a 15 min test session. One animal from the 2TR group was discarded because of equipment failure at the time of test. Thus, the 2TR group contained 6 subjects.

\section{Histology and Neurochemistry}

Two weeks after passive avoidance testing (i.e., 32 weeks after transplantation) all subjects were decapitated, their brains quickly and carefully removed from the cranium and stored at $-80^{\circ} \mathrm{C}$. Brains were sliced in a rostral-caudal direction on a cryostat microtome. Alternate 520 $\mu$ "thick" and $40 \mu$ "thin" sections were obtained up through the rostral third of the hippocampus. The "thin" sections were collected on individual microscopic slides and later stained for acetylcholinesterase histochemistry according to the general methods of Commins and Yahr /3/. The "thick" sections were mounted on precooled slides and the frontoparietal cortical areas dissected at $0^{\circ} \mathrm{C}$. Cortical pieces from each animal were pooled and frozen at $-80^{\circ} \mathrm{C}$ until assayed for choline acetyltransferase (CAT) and acetylcholinesterase (ACHE) according to the procedures described by Fonnum $/ 7 /$ and Johnson and Russell /10/, respectively.

\section{Statistical Analysis}

One-way ANOVAs followed by planned ttests were used to analyze all data except the results derived from passive avoidance. Because a large number of "ceiling" scores were obtained when subjects were tested on the passive avoidance task, nonparametric tests were used to analyze these data. Finally, since for the three water-motivated tasks, trials- (TTC) and errorsto-criterion (ETC) scores correlated (rs > .77, ps $<.01$ ) thus revealing similar patterns of results, only the TTC variable is presented.

\section{RESULTS}

\section{Complex Spatial Discrimination}

As is evident in Figure 1 , significant differences existed with respect to $\operatorname{TTC}[F(3,27)=$ $3.85, \mathrm{p}<.025]$.

Animals in the NBM group required on average more than twice the number of TTC than SHAM animals [ $\mathrm{t}(16)=2.44, \mathrm{p}<.05]$. This lesion-induced spatial memory impairment was attenuated by the transplantation manipulation as evidenced by the proficient performance of the 4TR group. Animals in the 4TR condition required less TTC than NBM animals [ $\mathrm{t}(13)=$ $1.83, \mathrm{p}<.05$, one-tailed] and were statistically comparable to SHAM animals $(p>.20)$. In contrast, animals in the 2TR group were as impaired as NBM subjects $(p>.20)$, requiring statistically more trials to reach criterion than SHAM subjects $[\mathrm{t}(14)=3.12, \mathrm{p}<.01]$. 
COMPLEX SPATIAL DISCRIMINATION

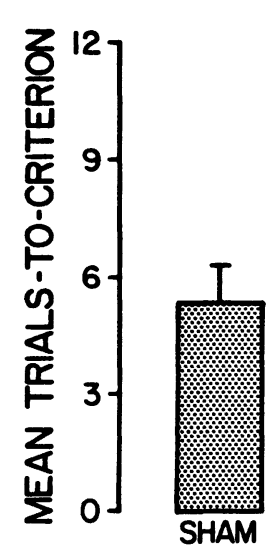

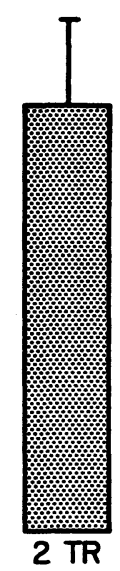

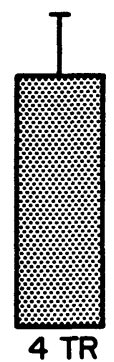

Fig. 1: Mean ( \pm SEM) trials-to-criterion associated with the four groups of subjects trained on the complex spatial discrimination task.

\section{Brightness Discrimination}

A significant overall difference between the groups was not obtained when the number of trials required to reach criterion was examined $(\mathrm{F}<1.0, \mathrm{p}>.20)$. It is interesting to note, however, that some evidence of transplant-induced improvement existed in that the 4TR group required significantly less ETC than NBM animals (mean ETC $=10.8$ vs. 21.1 , respectively) $[\mathrm{t}(13)=2.20, \mathrm{p}<.05]$. SHAM animals, on the other hand, committed an average of 14.3 ETC while subjects in the 2TR condition committed an average of 17.6 ETC. Neither the SHAM nor the 2TR group differed significantly from NBM animals $(\mathrm{ts}<1.20$, ps $>.20)$.

\section{Delayed Spatial Alternation}

As can be seen in Figure 2, groups differed with respect to TTC $[\mathrm{F}(\alpha) 3,23)=4.06, \mathrm{p}<$ .025].

Pairwise comparisons indicated that the NBM group required on average about three times the number of TTC than SHAM animals $[\mathrm{t}(12)=$ $3.39, \mathrm{p}<.01]$. As with the complex spatial discrimination task, transplants alleviated this lesion-induced memory impairment. However, in

contrast to the improved performance derived from the 4TR manipulation on the earlier task (see Fig. 1), the 2TR condition resulted in statistically better performance in the present test situation [2TR vs. NBM, $\mathrm{t}(13)=4.03, \mathrm{p}<$ .01]. Animals in the 4TR condition, although exhibiting a nominally lower mean TTC score, were not statistically different from NBM animals $(p>.10)$.

Analysis of percent correct performance revealed a pattern of results identical to that produced by the TTC measure. Of the 75 trials, NBM animals, on average, were correct on only $63.2 \%$ of the trials. This correct choice rate was significantly lower than the $72.2 \%$ mean rate exhibited by SHAM subjects $[\mathrm{t}(12)=3.29, \mathrm{p}<$ $.01]$ and the $71.8 \%$ mean rate associated with the 2TR group $[\mathrm{t}(13)=3.28, \mathrm{p}<.01]$. Rats in the 4TR condition on average were correct on $67.8 \%$ of the trials which did not differ from the percent correct rate of NBM animals ( $p>.20)$.

\section{Passive Avoidance}

The $72 \mathrm{hr}$ retention latencies for the four groups of subjects are presented in Table 1.

\section{TABLE 1.}

Median ( \pm Interquartile Ranges) 72 Hour Passive Avoidance Retention Latencies (sec).

\begin{tabular}{cccc}
\hline SHAM & NBM & 2 TR & 4 TR \\
\hline 900 & 900 & $204^{*}$ & 386 \\
$( \pm 570)$ & $( \pm 596)$ & $( \pm 236)$ & $( \pm 512)$ \\
\hline
\end{tabular}

* $\mathrm{p}<.05$ VS. SHAM and NBM, Mann-Whitney U test.

Analysis of these data by means of a KruskalWallis ANOVA corrected for ties indicated a significant overall effect $[\mathrm{H}(3)=7.38, \mathrm{p}=.06]$. Subsequent Mann-Whitney $U$ tests revealed significant differences between the 2TR group and both the SHAM $(U=9, p<.05)$ and NBM $(U=9, p<.05)$ groups. No other betweengroup differences in retention latencies existed. Finally, no differences between the groups were revealed when latencies on the training trial were examined $[\mathrm{F}(3,26)=0.85, \mathrm{p}>.20]$. 


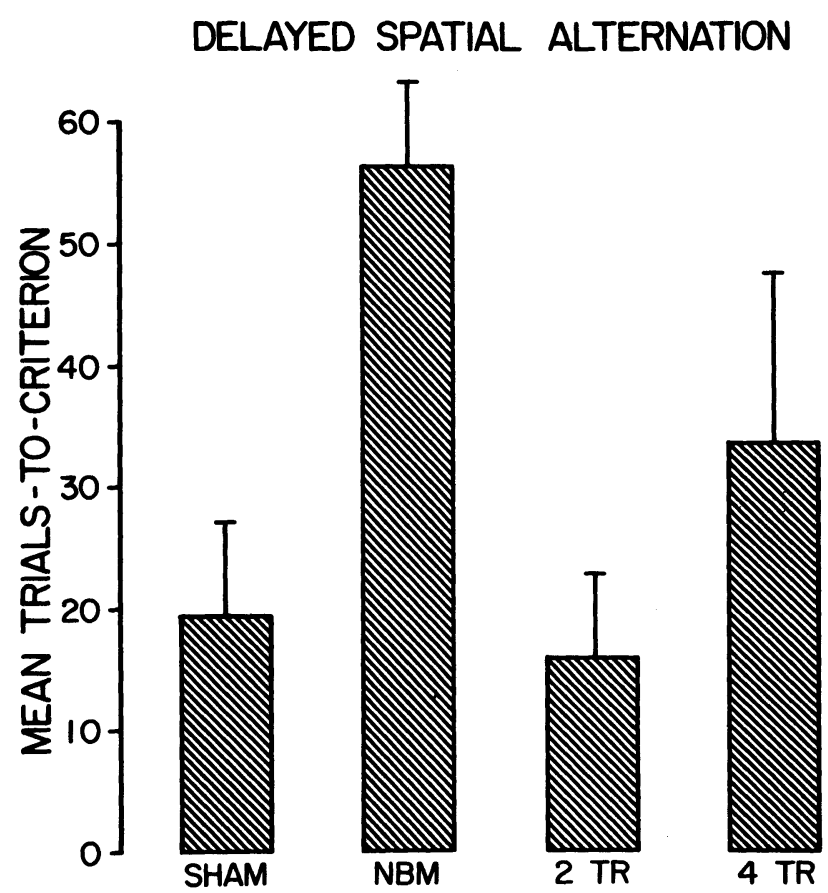

Fig. 2: Mean ( \pm SEM) trials-to-criterion associated with the four groups of subjects trained on the delayed spatial alternation task.

\section{Cortical CAT and ACHE Neurochemistry}

Separate ANOVAs performed on cortical $\mathrm{CAT}[\mathrm{F}(3,27)=4.02, \mathrm{p}<.025]$ and $\mathrm{ACHE}$ $[F(3,27)=4.09, p<.025]$ concentrations indicated significant differences between the groups (see Table 2).

Results indicated that NBM animals had lower concentrations of $\mathrm{CAT}$ and $\mathrm{ACHE}$ relative to SHAM subjects [ts $(16)>4.22$, ps $<.001$ ] This was in contrast to the levels found following transplantation. Both transplant conditions yielded concentrations that did not differ from SHAM values ( $\mathrm{ps}>.05)$. In fact, CAT $[\mathrm{t}(14)=$ $1.98, \mathrm{p}=.07]$ and $\mathrm{ACHE}[\mathrm{t}(14)=2.45, \mathrm{p}<.05]$ concentrations in 2TR animals were significantly greater than those values associated with NBM animals.

When cortical CAT and ACHE concentrations were correlated with performance on the complex spatial discrimination task, significant coefficients were obtained. Both TTC and ETC correlated negatively with cortical CAT (TTC: $\mathrm{r}=-.585, \mathrm{p}<.002$; ETC: $\mathrm{r}=-.618, \mathrm{p}<$
Table II.

Mean ( \pm SEM) concentrations of choline acetyltransferase (CAT) and acetylcholinesterase (ACHE) in the frontoparietal cortices. Values are expressed in $\mathrm{nmol} / \mathrm{hr} / \mathrm{mg}$ protein

\begin{tabular}{lcc}
\hline GROUP & CAT & ACHE \\
\hline SHAM & 57.6 & 2290 \\
& $( \pm 0.9)$ & $( \pm 43)$ \\
NBM & $* 49.0$ & $* 1896$ \\
& $( \pm 1.8)$ & $( \pm 87)$ \\
\% depletion & $-14.9 \%$ & $-17.2 \%$ \\
2TR & 54.5 & 2241 \\
& $( \pm 2.2)$ & $( \pm 115)$ \\
\% depletion & $-5.3 \%$ & $-2.1 \%$ \\
4TR & 53.4 & 2092 \\
& $( \pm 2.2)$ & $( \pm 105)$ \\
\% depletion & $-7.3 \%$ & $-8.6 \%$ \\
\hline
\end{tabular}

$\%$ depletion is relative to SHAM

* $\mathrm{p}<.001$, vs. SHAM, t-test

001) and ACHE (TTC: $\mathrm{r}=-460, \mathrm{p}<.02$; ETC: $\mathrm{r}=-.468, \mathrm{p}=.01)$ concentrations. Performance on no other task correlated significantly with cortical neurochemistry.

\section{Histology}

Histological analysis revealed a loss of ACHE-positive stained large cell in the area of the nbM following infusion of ibotenic acid. In addition, the lesion produced a less intense staining pattern in the frontoparietal cortex (see Figures $3 \mathrm{~A}$ and $3 \mathrm{~B}$ ).

Regardless of the number of implants, transplanted fetal material survived and exhibited very intense ACHE-positive staining pattern. In addition, fetal transplants at 2 and at 4 cortical sites normalized the abnormal cortical staining pattern seen following nbM lesions, at least in the areas around the transplantation sites (see Figures 4A and 4B).

\section{DISCUSSION}

The present data contribute further to the existing literature suggesting that transplants 

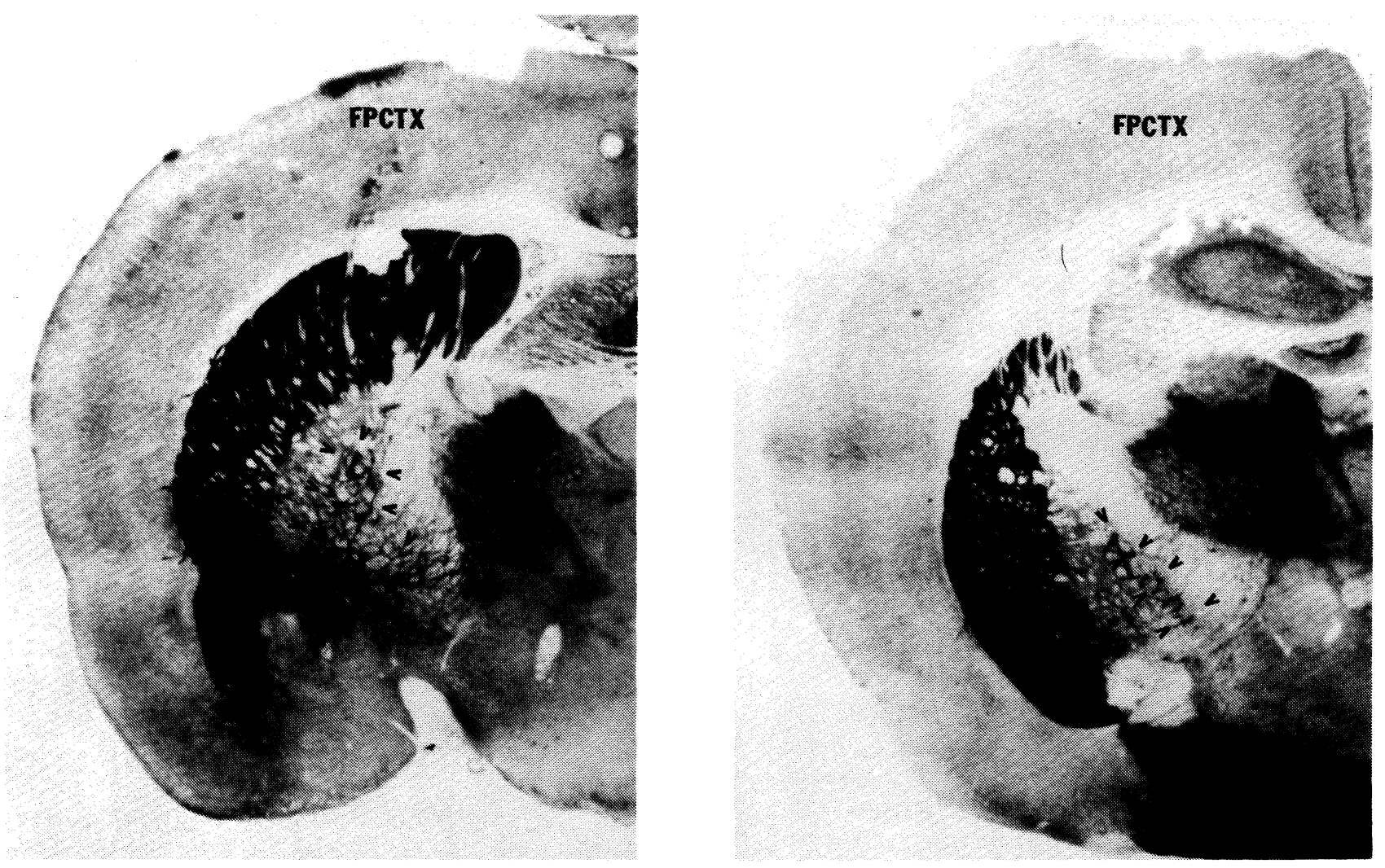

Figs. 3A and B: Representative coronal sections through the $\mathrm{nbM}$, stalned for ACHE histochemistry, from a sham (A) and a nbM-lesioned (B) subject. Note the paucity of ACHE-positive stained cells (at the arrowheads) in the nbM-lesioned animal relative to the number of cells in the sham subject. Also note the less intense staining pattern associated with the dorsolateral aspect of the frontoparietal cortices (FPCTC) in the nbM-lesioned animal.

derived from cholinergic-rich, fetal ventral forebrain tissue can indeed reverse some of the memory and neurochemical abnormalities produced by lesions of the nbM-frontal cortical system in rats. However, the exact transplant parameters, specifically the number of implantation sites, necessary for recovery of memory function appear to depend upon the cognitive demands of the behavioral task and/or the length of time since transplantation. This is illustrated by the results derived from the complex spatial discrimination and the delayed spatial alternation tasks. Animals with nbM lesions and transplants at 4 cortical locations performed better than lesion-alone subjects on the former task, while the 2 transplant condition yielded better performance on the latter task.

One obvious explanation that can be suggested to account for such a result has to do with the memory demands of each task. Although both tasks are "spatial" in nature, the fact that the delayed spatial alternation task requires greater demands on working-memory might account for the differential influence of 2 vs. 4 transplants. That is, successful performance on the alternation task requires animals to remember, during the $30 \mathrm{sec}$ delay period, which arm they visited last. In contrast, no comparable "trial-dependent" information needs to be remembered when subjects are trained on the complex spatial discrimination task. The fact that the 4-site condition yielded some evidence of memory enhancement on the brightness discrimination task (which, like the complex spatial discrimination task, does not tax workingmemory capabilities) is consistent with the notion that cognitive requirements of the task play an important role in determining which 

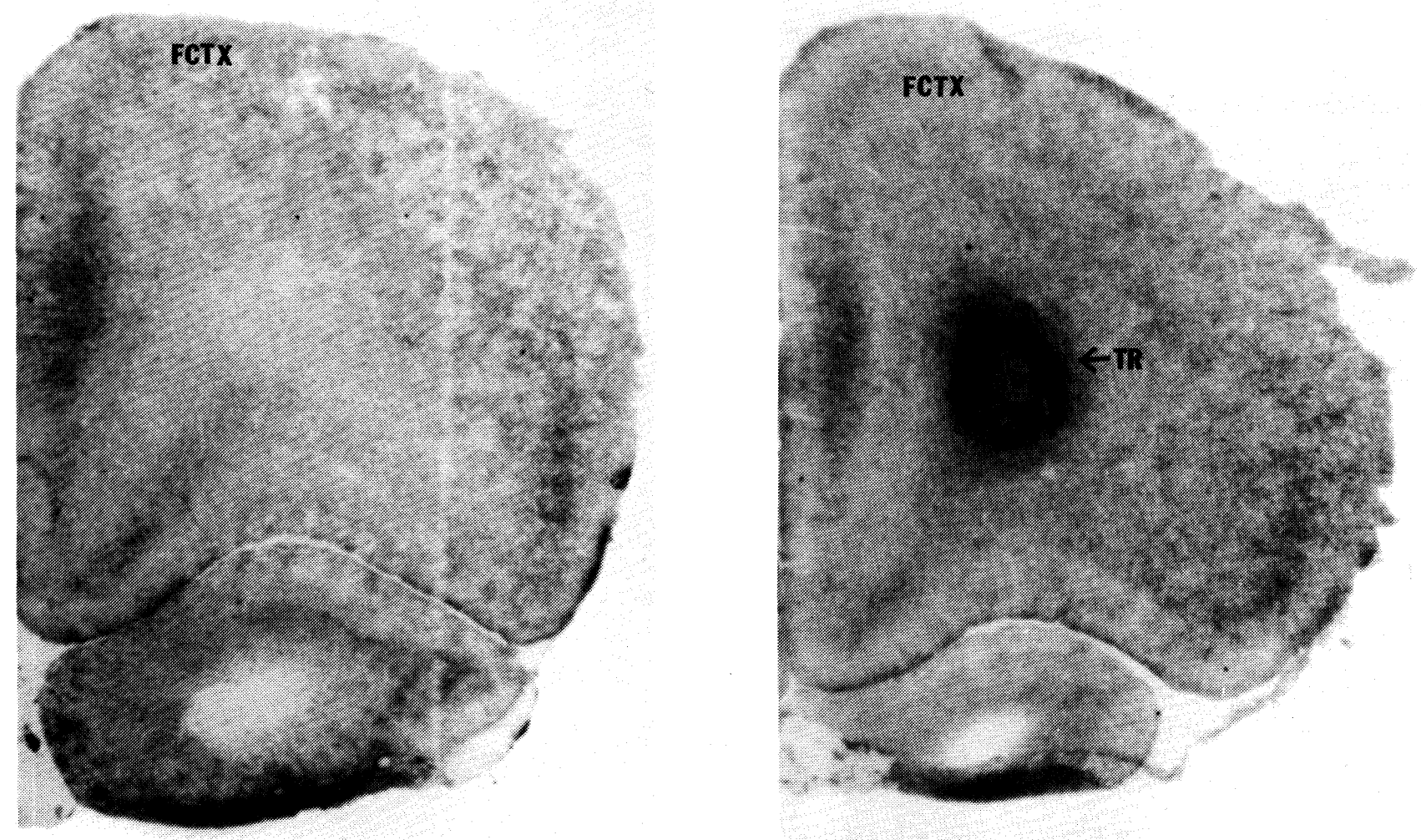

Figs. 4A and B: Representative coronal sections through the rostral frontal cortex (FCTX), stained for ACHE histochemistry, from a nbM-lesioned (A) and a 2-site transplant (B) animal. The staining pattern in the FCTX is less intense in the nbM-lesioned animal relative to the typically extensive staining pattern associated with sham animals (not shown). The darkly stained central portion within the FCTX of the transplant subject is the fetal graph (TR). Note that the transplanted tissue not only survived but was associated with an increased staining intensity pattern in the surrounding FCTX.

transplant manipulation yields reversal of lesioninduced memory impairments.

A second, perhaps more parsimonious, interpretation to explain the differential effects of 2 vs. 4 transplants is related simply to time following transplantation. Assuming that the complex spatial discrimination and the delayed spatial alternation tasks are fairly comparable in difficulty, it is reasonable to suggest that the 4site manipulation is effective early on (at about 3 - 4 months following transplantation) while the 2site condition is effective later on (at about $6-7$ months following transplantation). If such a hypothesis were true, then it could be speculated that an optimal number of connections between the transplant and host needs to be formed in order for lesion-induced impairments in memory to be alleviated. Thus, since more tissue is transplanted, the 4 transplant condition would require a shorter amount of time before recovery was evident. However, this recovery would wane over time with the formation of an excessive (i.e., more than optimal) number of connections. In contrast, the 2 transplant condition would need more time before a sufficient amount of connections that would support memory recovery are formed. It is interesting to note that posttransplantation time has been reported to be important in determining whether animals with medial frontal cortical lesions exhibited recovery on the Morris water maze spatial navigation task /11/. Unfortunately, the present data cannot discriminate between these two mechanisms or other possible mechanisms (e.g., neurotrophic substances) responsible for the differential effects of the 2- and 4-site transplant conditions. 
Regardless of the exact mechanism responsible for recovery, the fact that fetal transplants did indeed reverse bilateral nbM lesion-induced memory deficits, extends the generalizability of transplant-induced memory recovery /4/. Furthermore, the findings of the present investigation also are consistent with a recently published report. In that study cholinergic-rich fetal transplants were demonstrated effective in ameliorating retention deficits produced by bilateral lesions of the nbM when subjects were trained on a delayed spatial alternation task similar to the one used here $/ 16 /$. Also consistent with the present results are data reported by Arendash and Mouton /1/ showing a failure of fetal transplants to enhance long-term retention (24 and $48 \mathrm{hr}$ ) of a passive avoidance response. Such a result is reminiscent of the present $72-\mathrm{hr}$ passive avoidance retention findings and suggests that not all tests of memory are equally affected by fetal transplantation. However, it should be noted that 8 implantation sites (4/hemisphere, 2 $\mu l$ each) were employed in the Arendash and Mouton investigation while a long posttransplantation interval (7.5 months) had elapsed before subjects were trained on passive avoidance in the present study. Thus the possibility exists that transplantation of too much fetal material or waiting too long before training might account for the lack of behavioral enhancement of passive avoidance.

Data derived from the neurochemical analyses correspond with the behavioral results. It was revealed that, although both transplant groups exhibited concentrations of CAT and ACHE that were statistically equivalent to the values associated with sham operated subjects, only animals in the 2TR group showed significantly greater concentrations than those found in nbM-lesioned subjects. This is interesting considering that the 2TR group was the condition that yielded memory recovery most recently prior to sacrifice (i.e., on the delayed spatial alternation task). It is also interesting to note that CAT and ACHE levels significantly correlated with performance on the complex spatial discrimination task. This result suggests that successful performance on this task is highly dependent upon the integrity of the basal forebrain-frontal cortical cholinergic system and encourages further use of this task in studies of nbM function.
In conclusion, the present study clearly illustrates the effectiveness of fetal transplants in reversing those memory and neurochemical impairments following lesions of the nbM in rats. However, the exact transplant parameters necessary for such recovery are dependent upon the particular assessment instrument used and/or the posttransplantation interval.

\section{ACKNOWLEDGEMENTS}

The authors thank John Bangston and Renee Gluck for their help in this investigation. A preliminary report of these data was presented at the 1988 meeting of the Society for Neuroscience, Toronto, Canada. The research reported in this communication was supported by a VA Merit Review grant awarded to Kenneth L. Davis and Vahram Haroutunian.

\section{REFERENCES}

1. Arendash GW, Mouton PR. Transplantation of nucleus basalis magnocellularis cholinergic neurons into the cholinergic-depleted cerebral cortex. Annals NY Acad Sci 1987; 495; 431-443.

2. Bjorklund A, Stenevi U, Schmidt RH, Dunnett SB, Gage, FH. Intracerebral grafting of neuronal cell suspensions. I. Introduction and general methods of preparation. Acta Physiol Scand (Suppl) 1983; 522: 17.

3. Commins D, Yahr P. Adult testosterone levels influence the morphology of a sexually dimorphic area in the Mongolian gerbil brain. J Comp Neurol 1984; 224: 132-140.

4. Dunnett SB, Toniolo G, Fine A, Ryan CN, Bjorklund $A$, Iversen SD. Transplantation of embryonic ventral forebrain neurons to the neocortex of rats with lesions of the nucleus basalis magnocellularis - II. Sensorimotor and learning impairments. Neuroscience 1985; 16: 787-797.

5. Dunnett SB, Whishaw IQ, Bunch ST, Fine A. Acetylcholine-rich neuronal grafts in the forebrain of rats: Effects of environmental enrichment, neonatal noradrenaline depletion, host transplantation site and regional source of embryonic donor cells on graft size and acetylcholinesterase-positive fibre outgrowth. Brain Res 1986; 378: 357-373.

6. Fine A, Dunnett SB, Bjorklund A, Clarke D, Iversen SD. Transplantation of embryonic ventral forebrain neurons to the neocortex of rats with lesions of nucleus basalis magnocellularis - I. Biochemical and anatomical observations. Neuroscience 1985; 4: 769 . 786. 
7. Fonnum F. A rapid radiochemical method for the detection of choline acetyltransferase. J Neurochem 1975; 24: 407-408.

8. Gash DM. Neural transplantation: Potential therapy for Alzheimer's disease. J Neural Trans (Suppl) 1987; 24: 301-308.

9. Gash DM, Collier TJ, Sladek JR. Neural transplantation: A review of recent developments and potential applications to the aged brain. Neurobiol of Aging 1985; 6: 131-150.

10. Johnson $C D$, Russell $R L$. A rapid, simple radiometric assay for acetylcholinesterase, suitable for multiple determinations. Annals Biochem 1975; 26: 229-232.

11. Kolb B, Reynolds B, Fantie B. Frontal cortex grafts have opposite effects at different postoperative recovery times. Behav and Neural Biol 1988; 50: 193206.

12. Murray CL, Fibiger HC., Pilocarpine and physostigmine attenuate spatial memory impairments produced by lesions of the nucleus basalis magnocellularis. Behav Neurosci 1986; 100: 23-32.
13. Santucci AC, Haroutunian V. Nucleus basalis lesions impair memory in rats trained on nonspatial and spatial discrimination tasks. Physiol and Behav 1989; 45: 1025-1031.

14. Santucci AC, Haroutunian V, Bierer LM, Davis KL Approaches to the treatment of Alzheimer's disease. In: Lister $\mathrm{R}$, Weingartner $\mathrm{H}$ (eds.), Perspectives in Cognitive Neuroscience. Oxford: Oxford University Press, in press.

15. Santucci AC, Kanof PD, Haroutunian V. Effect of physostigmine on memory consolidation and retrieval processes in intact and nucleus basalis-lesioned rats. Psychopharmacology 1989; 99: 70-74.

16. Welner SA, Dunnett SB, MacLean B, Iversen SD., Transplantation of embryonic ventral forebrain grafts to the neocortex of rats with bilateral lesions of nucleus basalis magnocellularis ameliorates a lesioninduced deficit in spatial memory. Brain Res 1988; 463: 192-197.

17. Wenk G, Hughey D, Boundy V, Kim A, Walker L, Olton D. Neurotransmitters and memory: Role of cholinergic, serotonergic and noradrenergic systems. Behav Neurosci 1987; 101: 325-332. 

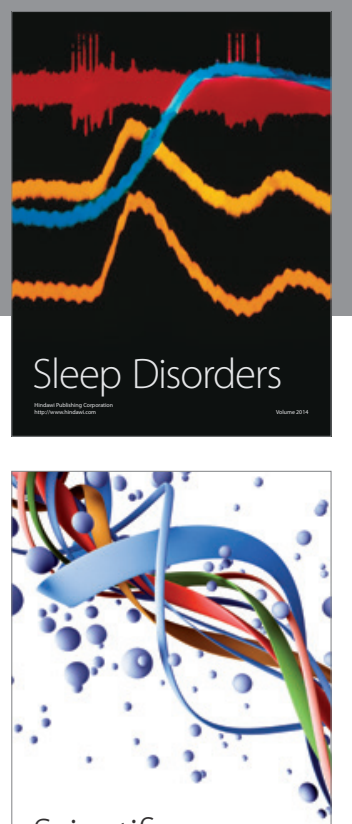

Scientifica
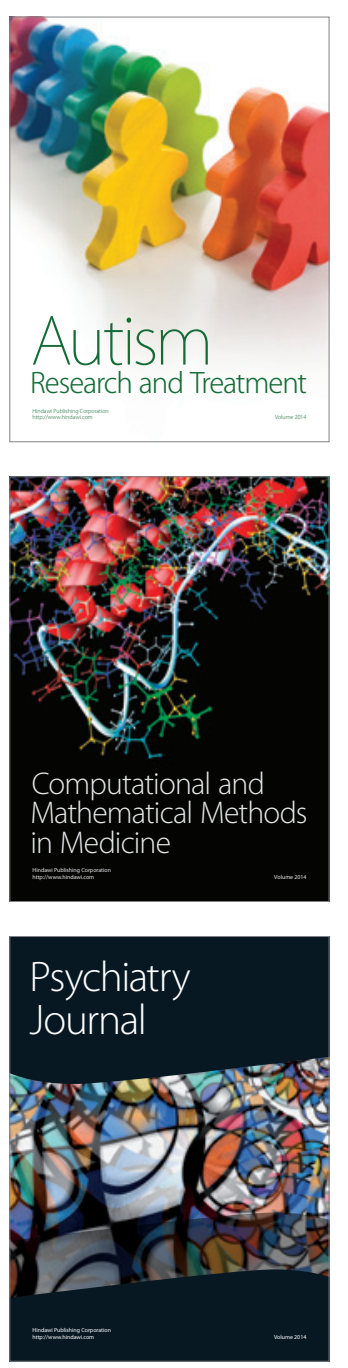
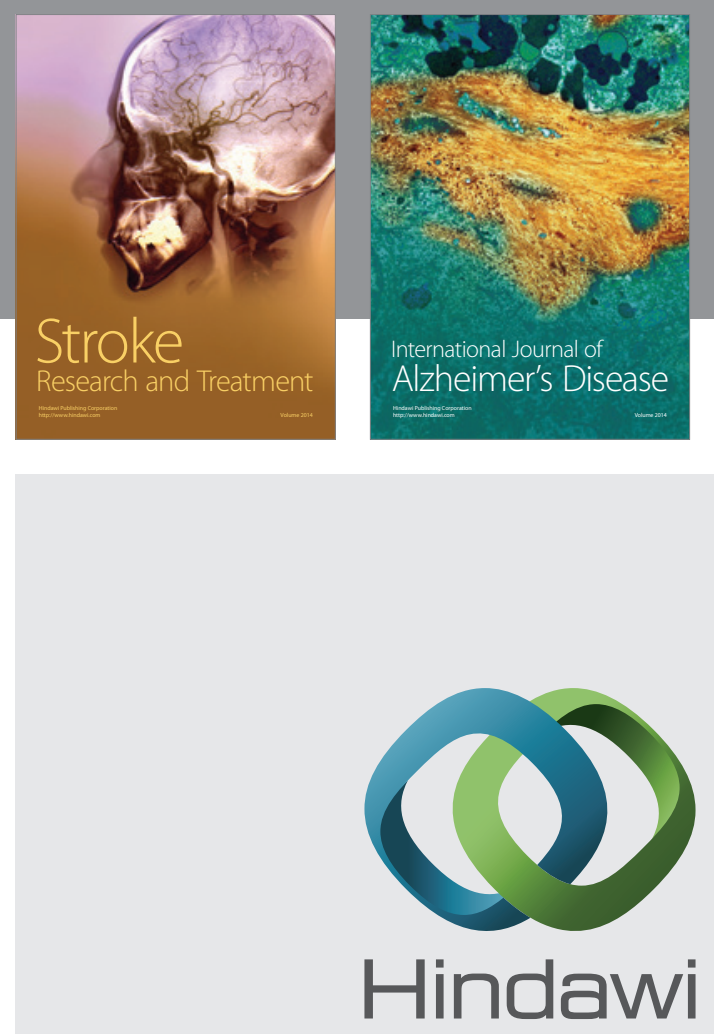

Submit your manuscripts at

http://www.hindawi.com
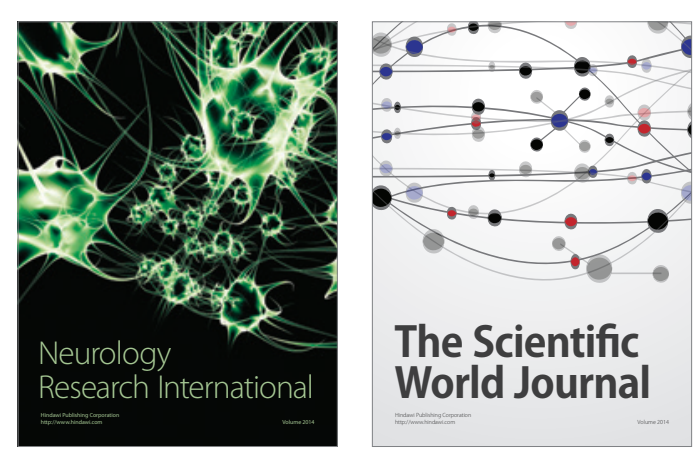

The Scientific World Journal

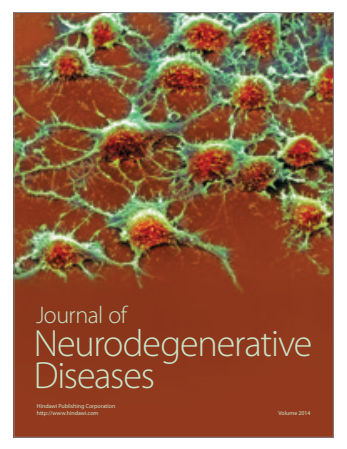

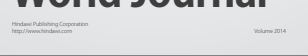

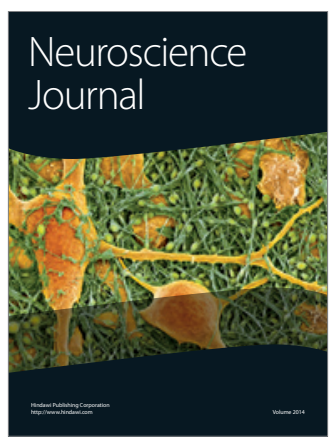

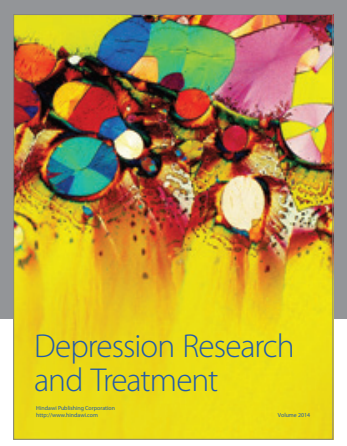
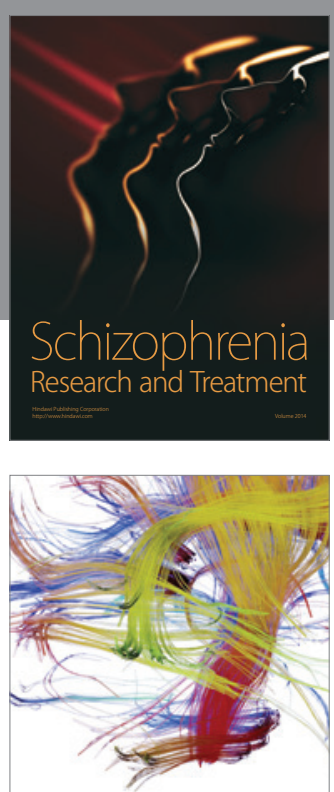

Brain Science

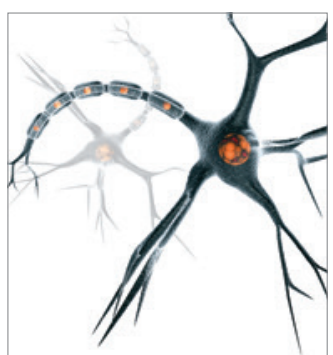

Neural Plasticity
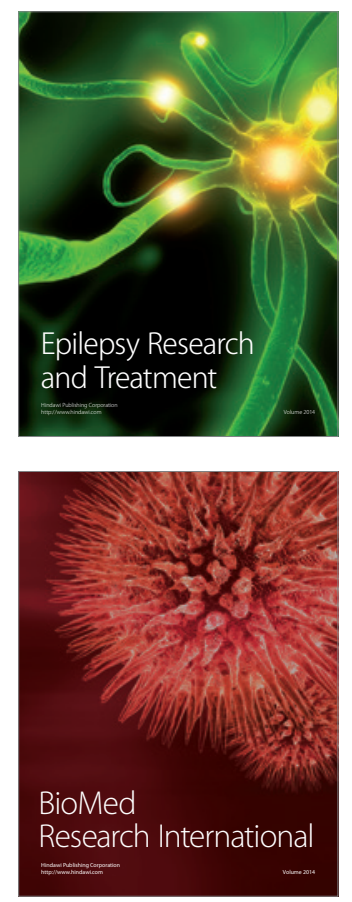

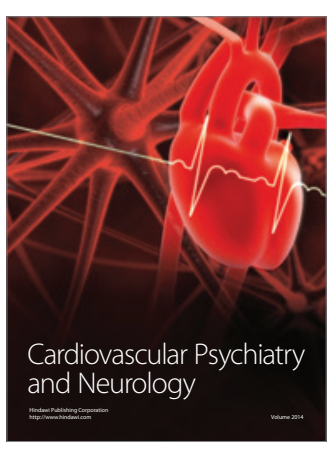

Parkinson's

Disease
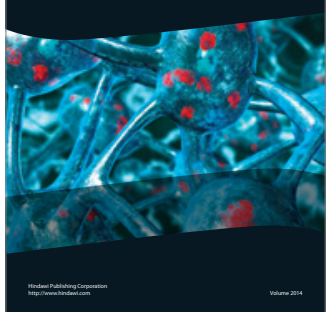